\title{
Detection of cyclic nucleotide phosphodiesterase mRNA in mouse skeletal muscle tissue and primary cultured myocytes
}

\author{
J.N. Gu' ${ }^{1}$ W. Chen ${ }^{2}$, T.Q. Yu ${ }^{3}$, P. Lu ${ }^{3}$, X. Mu ${ }^{2}$ and J.Q. Xu ${ }^{1,4}$ \\ ${ }^{1}$ College of Veterinary Medicine, China Agricultural University \\ 100094, Beijing, P.R. China
}

${ }^{2}$ Key Laboratory of Traditional Chinese Veterinary Medicine, Department of Animal Science and Technology, Beijing University of Agriculture 102206, Beijing, P.R. China

${ }^{3}$ Key Laboratory of New Technology in Agriculture Application, Beijing University of Agriculture 102206, Beijing, P.R. China

(Received 10 January 2007; revised version 17 July 2007; accepted 21 November 2007)

\begin{abstract}
The regulation of postnatal skeletal muscle development is complex, involving many integrated biochemical pathways that interact with the environment to ultimately control the rate of protein accretion. The intracellular level of cyclic nucleotides (cAMP/cGMP), which are predominately regulated by phosphodiesterase (PDE), is important for skeletal muscle protein accumulation. To determine whether PDE might play a potential role in postnatal skeletal muscle development, eighteen pairs of specific primers were designed for determining different PDE isozyme mRNA in skeletal muscle tissue and primary cultured myocytes by using RT-PCR. The results showed that seventeen PDE subtypes except PDE8B were expressed at the transcriptional level, with PDE4A, 4B, PDE7A, and PDE9A subtypes displaying stronger expression than others. This is the first report that 18 subtypes of PDE isozymes are expressed in skeletal muscle tissue and primary cultured myocytes, thus implicating their role in postnatal skeletal muscle development.
\end{abstract}

KEY WORDS: phosphodiesterase isozymes, gene expression, skeletal muscle, primary cultured myocytes

${ }^{1}$ Corresponding author: e-mail: jianqin-xu@hotmail.com

The authors wish it to be known that, in their opinion, the first two authors should be regarded as joint First Authors 


\section{INTRODUCTION}

The regulation of postnatal skeletal muscle development is complex, involving many integrated biochemical pathways that interact with the environment to ultimately control the rate of protein accretion. Cyclic adenosine 3', 5'-monophosphate (cAMP) and cyclic guanosine 3', 5'-monophosphate (cGMP), which act as second messengers, play a crucial role in cell replication, differentiation, and in the function of a terminally differentiated cell in various tissues (Dumont et al., 1989; Shaulsky et al., 1998). It is well established that cAMP regulates a wide variety of skeletal muscle physiological processes, mediating the biological action of many extracellular substances. It has been reported that increased intracellular levels of cAMP and cGMP, respectively, inhibit and stimulate the skeletal muscle proteolysis rate and the synthesis and release of the amino acids, alanine and glutamine (Garber, 1978; Garber et al., 1980).

The cAMP- or cGMP-mediated intracellular signaling is controlled not only by cyclic nucleotide generation in response to extracellular stimulation, but also by the PDE-mediated hydrolysis of the cyclic nucleotide (Soderling and Beavo, 2000). Hydrolysis of the cyclic nucleotides occurs at a rate 9-600-fold faster than the rate of cyclic nucleotide synthesis, suggesting that PDE plays the dominant role in the control of cyclic nucleotide levels in cells (Cheng and Boettcher, 1982). At least 11 different PDE families (PDE1 to PDE11) have been identified in mammalian tissues on the basis of their amino-acid sequence homology and biochemical properties, including substrate specificity, stimulation or inhibition by endogenous regulators, and sensitivity to PDE inhibitors (Soderling and Beavo, 2000; Bingham et al., 2006). Of these eleven families, rolipram-sensitive PDE4, rolipram-insensitive PDE7 and PDE8 isozymes hydrolyse mainly cAMP. PDE5, photoreceptor PDE6 and PDE9 isozymes prefer cGMP as a substrate. Calcium/calmodulin-dependent PPDE1, cGMP-stimulated PDE2, cGMP-inhibited PDE3, PDE10 and PDE11 are dual-substrate PDEs that hydrolyse both cAMP and cGMP; however, PDE3 hydrolyses cAMP 10 times faster than cGMP. Subfamilies/subtypes encoded by distinct genes are found in the PDE1, PDE3, PDE4, PDE6, PDE7, and PDE8 families. In many cases, these gene products show unique tissue expression patterns and subcellular localizations, distinct biochemical properties, specific regulations mediated by some kinases, including cAMP-dependent protein kinase and cGMP-dependent protein kinase and association with other proteins (Soderling and Beavo, 2000). Therefore, PDEs comprise a superfamily, and control cyclic nucleotide levels.

Several diseases linked to defects in PDE function have been described, and there has been a growing interest in the use of isozyme-specific PDE inhibitors as pharmacological agents to treat a number of different health disorders. The role of PDEs in skeletal muscle tissue and myocytes has surprisingly attracted little atten- 
tion, despite the cyclic nucleotides being known to participate in the signaling of a variety of important events in skeletal muscle biology, such as protein metabolism. Most mammalian tissues express several members of the PDE family and many express more than one subtype of an individual family (Manganiello et al., 1995). There are, however, numerous examples when an individual PDE is found predominantly in a specific localization (Kuthe et al., 2001). In our study, we used the mouse as a model to investigate the expression profile of PDE mRNAs in skeletal muscle tissue and primary cultured myocytes.

\section{MATERIAL AND METHODS}

\section{Cell cultures}

Primary cultured myocytes were prepared from skeletal muscle as described previously with minor modification (Hellsten and Frandsen, 1997). One-to three-day-old newborn ICR mice were killed by cervical dislocation, and muscle tissue from limbs was dissected followed by digestion with $0.1 \%(\mathrm{w} / \mathrm{v})$ collagenase II (Sigma-Aldrich, St. Louis, Missouri, USA) and $0.2 \%(\mathrm{w} / \mathrm{v})$ trypsin (GIBCO Lab., Grand Island, New York, USA) in $5 \mathrm{ml}$ Hank's balance salt solution (Sigma-Aldrich, St. Louis, Missouri, USA) at $37^{\circ} \mathrm{C}$ for $30 \mathrm{~min}$. Dulbecco's modified Eagle's medium (DMEM) (Gibco Lab., Grand Island, New York, USA) containing 15\% (v/v) foetal calf serum (FCS) (Gibco Lab., Grand Island, New York, USA) and $100 \mathrm{U} / \mathrm{ml}$ penicillin/streptomycin was added (growth medium): the remaining clumps were dissociated by trituration with a $5 \mathrm{ml}$ pipette. The cell suspension was centrifuged at $200 \mathrm{~g}$ for $10 \mathrm{~min}$ and the supernatant was discarded. Ten milliliters of growth medium were added and the suspension was again triturated with a $5 \mathrm{ml}$ wide bore pipette to dissociate aggregated cells. The suspension was filtered through $100 \mu \mathrm{m}$ nylon mesh and the cells were seeded out onto two $60 \mathrm{~mm}$ Petri dishes for $60 \mathrm{~min}$ to allow fibroblasts to attach to the bottom, whereas myoblasts remained in suspension. The medium was removed from the dishes and the dishes were discarded. Cells were counted and seeded out onto 6-well culture plates coated with $0.1 \%(\mathrm{w} / \mathrm{v})$ gelatin at a density of $2-5 \times 10^{5}$ cells per well. The cells were incubated at $37^{\circ} \mathrm{C}$ in an incubator with water-saturated mixture of $5 \% \mathrm{CO}_{2}$ and $95 \%$ air. After $48 \mathrm{~h}$, the media was changed to a fresh one with $10 \%$ FCS. To induce cell differentiation and myotube fusion the medium was changed to a fusion medium containing 5\% FCS and $2 \mathrm{mM}$ L-glutamine on day 4 after plating. The experiments were performed after myotubes formed and cross-striations and spontaneous twitching became apparent on the 6-7th day. 


\section{Isolation of total RNA and reverse transcription}

Skeletal muscle strips and primary cultured myocytes were used for extracting total RNA. The skeletal muscle strips were immediately cut from healthy adult male ICR mouse limbs after killing by cervical dislocation and were shock-frozen in liquid nitrogen. Myocytes, grown on 6-well culture plates, were washed twice with phosphate-buffered saline (PBS) without RNase. Total RNA was isolated using RNAgent ${ }^{\circledR}$ Total RNA Isolation System (Promega, Madison, Wisconsin, USA) according to the manufacturer's instructions. Extracted RNA was quantified spectrophotometrically. Subsequently, $1 \mu \mathrm{g}$ of total RNA as a template was reversetranscribed into single-stranded cDNA using oligo $(\mathrm{dT})_{16}$ primers and Moloney murine leukaemia virus reverse transcriptase (M-MLV RT) (Promega, Madison, Wisconsin, USA) according to the manufacturer's instructions in a final volume of $25 \mu \mathrm{l}$. The reaction was performed at $37^{\circ} \mathrm{C}$ for $60 \mathrm{~min}$. The reaction mixture containing the cDNA was then stored at $-20^{\circ} \mathrm{C}$.

\section{PCR amplification}

PCR reactions were performed using the following conditions: $1 \mu 110 \mu \mathrm{M}$ of each forward and reverse primer; $2 \mu \mathrm{l}$ of $5 \times$ PCR Buffer; $2 \mu 125 \mathrm{mM}$ of $\mathrm{MgCl}_{2} ; 2 \mu \mathrm{l}$ $2.5 \mathrm{mM}$ of dNTP; $0.8 \mathrm{U}$ of Taq Polymerase (Promega, Madison, Wisconsin, USA); $1 \mu \mathrm{l}$ of the cDNA synthesis reaction; nuclease-free water to a total volume of $20 \mu$. For each pair of primers, the annealing temperature was determined by performing gradient PCR from 55.0 to $64.0^{\circ} \mathrm{C}$ to find the optimal temperature. The specific primer sequences and their annealing temperature and product sizes are listed in Table 1. Amplifications were performed on a BIO-RAD PTC-200 Thermo Cyclers using the following program: $94^{\circ} \mathrm{C}$ for $5 \mathrm{~min} ; 94^{\circ} \mathrm{C}$ for $30 \mathrm{sec}$, annealing for $40 \mathrm{sec}$, $72^{\circ} \mathrm{C}$ for $30 \mathrm{sec}$, for $35 \mathrm{cycles}$; a final extension of $72^{\circ} \mathrm{C}$ for $8 \mathrm{~min}$. Otherwise, the cDNA reaction mixture from primary cultured myocytes was diluted 1:50, 1:100, and 1:1000 with nuclease-free water for other PCR amplifications. For analysis of the amplification products, electrophoresis was performed on $1.5 \%(\mathrm{w} / \mathrm{v})$ agarose gels, stained with ethidium bromide, and photographed under UV light.

Eighteen PDE subtype specific primer sequences were designed according to the complementary (c) DNA sequences available from GenBank databases, except PDE6A-6D, which are photoreceptor PDE. The glyceraldehyde-3- phosphate dehydrogenase (GAPDH) gene was used as the control reference.

\section{RESULTS}

The $A_{260} / A_{280}$ ratios of RNA samples from skeletal muscle tissue and primary cultured myocytes were 1.89 and 1.96 , respectively. The RNA samples were tested 
Table 1. Primer sequences, product size and PCR annealing temperature

\begin{tabular}{|c|c|c|c|}
\hline Subtype & Sequence & $\begin{array}{l}\text { Product } \\
\text { size }(b p)\end{array}$ & $\begin{array}{c}\text { Annealing } \\
\text { temperature } \\
{ }^{\circ} \mathrm{C}\end{array}$ \\
\hline PDE1A F & CACTGGCTCACTGAACTG & & \\
\hline PDE1A R & TGC(G/A)TGGAG(A/T)ATCAGGGAC & 369 & 59.8 \\
\hline PDE1B F & CCTTACCACAACCAGATCC & & \\
\hline PDE1B R & CATCTC(G/A)TC(G/A)TCCTGCATCA & 267 & 63.0 \\
\hline PDE1C F & GGT(A/G)ATGGCCACAGATATGTC & & \\
\hline PDE1C R & ACTTTCGGTCACACAGAGGAG & 251 & 56.6 \\
\hline PDE2A F & GTTCCTGCTGGATCAGAATG & & \\
\hline PDE2A R & ATGCC(G/A)CAGTAGATGGAGAAG & 342 & 64.0 \\
\hline PDE3AF & CTGGACAAACCAATTCTTGC & & \\
\hline PDE3A R & A(T/G)CC(A/G)ATCTCCAAAGCATG & 238 & 59.8 \\
\hline PDE3B F & AGGAGGTCCAGCTGCGTGTC & & \\
\hline PDE3B R & TTTGGCCTACAGGAACCTGAGA & 314 & 60.6 \\
\hline PDE4A F & $(\mathrm{A} / \mathrm{G}) \mathrm{GA}(\mathrm{G} / \mathrm{A}) \mathrm{GA}(\mathrm{C} / \mathrm{G}) \mathrm{AACTGCGACATC}$ & & \\
\hline PDE4A R & C(A/G)CCCTGCTG(G/A)AAGAACTC & 308 & 60.6 \\
\hline PDE4B F & TGGAAGACCTGAACAAATG & & \\
\hline PDE4B R & ACCAT(T/C)TTCCTGAGTGTCTG & 505 & 60.6 \\
\hline PDE4C F & GTCCAAACACATGAACCTCC & & \\
\hline PDE4C R & GTAGTCAATGAAACCCACCTG & 307 & 60.6 \\
\hline PDE4D F & (T/C)CTCTCTGAAATGAGTCG & & \\
\hline PDE4D R & CAC(A/G)TCTTCTAGTTCCTTGG & 251 & 56.6 \\
\hline PDE5A F & TTGACCG(A/G)CCTCTTAGACCC & & \\
\hline PDE5A R & CTTTGATGTTCAAGGGCTC & 414 & 56.6 \\
\hline PDE7A F & GA(T/C)GTTACTCAGGCCATGC & & \\
\hline PDE7A R & CCTCCGTTACTTTTTCACTCC & 493 & 60.6 \\
\hline PDE7B F & GGT(C/G)AACCAGCCATTTTTG & & \\
\hline PDE7B R & TC(T/C)TTATTGTGGAGGTGAGC & 249 & 60.6 \\
\hline PDE8A F & TGACACTGCTGTGCTGGAGAG & & \\
\hline PDE8A R & GTTGCCAAGGGTTTGTTGATG & 210 & 60.6 \\
\hline PDE8B F & TAAGTGTGCTGACGTGGC & & \\
\hline PDE8B R & (C/T)TCT(G/C)TGGCTTGGCTTTAG & 343 & 60.6 \\
\hline PDE9A F & GT(G/C)CGCTACAA(C/T)GACATCTC & & \\
\hline PDE9A R & TGGTCACTTTGTCTCGGTCC & 409 & 61.8 \\
\hline PDE10A F & TGGGCTTC(A/C)GTAGCAATAC & & \\
\hline PDE10A R & GCACCAC(A/G)CC(T/G)ATCACGCTG & 462 & 62.8 \\
\hline PDE11A F & GAACCAACAATGCCTTCCAAG & & \\
\hline PDE11A R & GGTCACGGCTCCAAGGTCAC & 335 & 63.4 \\
\hline GAPDH F & $\mathrm{C}(\mathrm{A} / \mathrm{C}) \mathrm{TCCTG}(\mathrm{C} / \mathrm{T}) \mathrm{ACCACCAACTG}$ & & \\
\hline GAPDH R & $\mathrm{G}(\mathrm{G} / \mathrm{C})(\mathrm{G} / \mathrm{A}) \mathrm{GT}(\mathrm{A} / \mathrm{G}) \mathrm{GG}(\mathrm{A} / \mathrm{G})$ ACACGGAAG & 265 & 64.0 \\
\hline
\end{tabular}


(a)
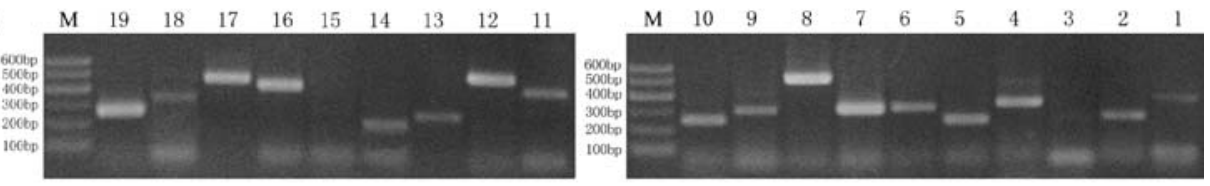

(b)
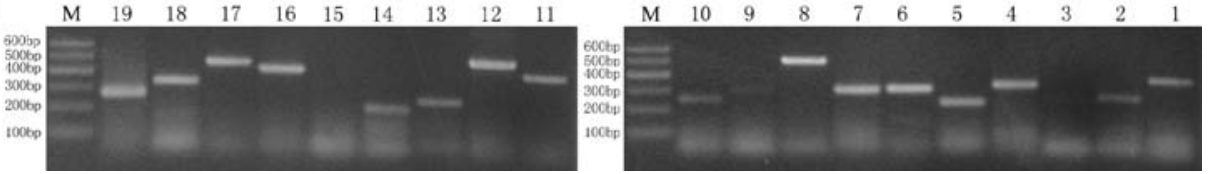

Figure 1. Different PDE subtypes mRNA was detected in skeletal muscle tissue (a) and primary cultured myocytes (b). Lanes from 1 to 18 correspond to PDE1A, 1B, 1C, PDE2A, PDE3A, 3B, PDE4A, 4B, 4C, 4D, PDE5A, PDE7A, 7B, PDE8A, 8B, PDE9A, 10A, and PDE11A; lane 19 corresponds to control GAPDH. Lane $\mathrm{M}$ is molecular weight markers

by PCR without prior reverse transcription to be sure that the PCR signals were not caused by contaminating DNA.

To verify the identity of PCR products, PDE2A, PDE4A, 4B, 4C, 4D, PDE7B and PDE11A subtypes, which were amplified from pig tissues in our earlier experiment, were chosen to be sequenced. Alignment of all resulting sequences with published sequence information confirmed that the PCR amplification products were PDE-specific. The seven above PCR product sequencing results were submitted to GenBank and have been accepted and released (accession numbers PDE2A: EF109890; PDE4A: DQ985705; PDE4B: DQ990376; PDE4C: EF109892; PDE4D: EF109889; PDE7B: EF109891; PDE11A: EF109893).
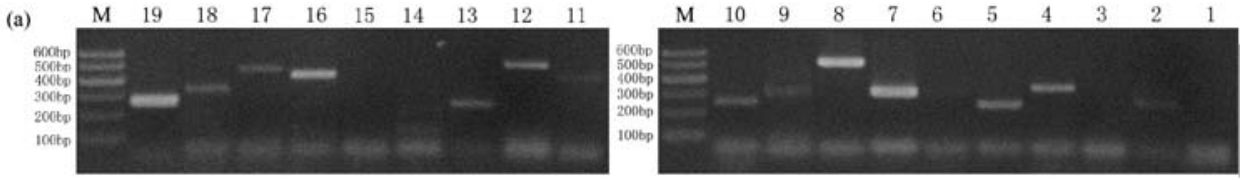

(b)
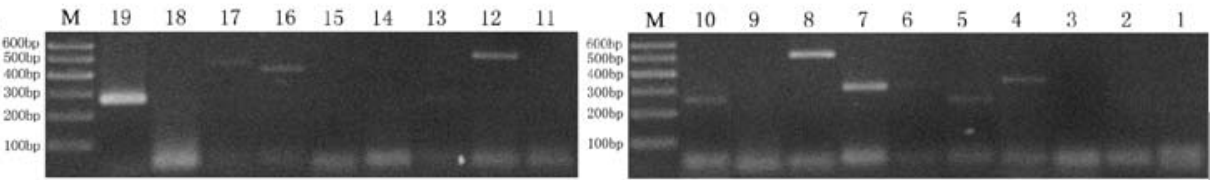

(c)
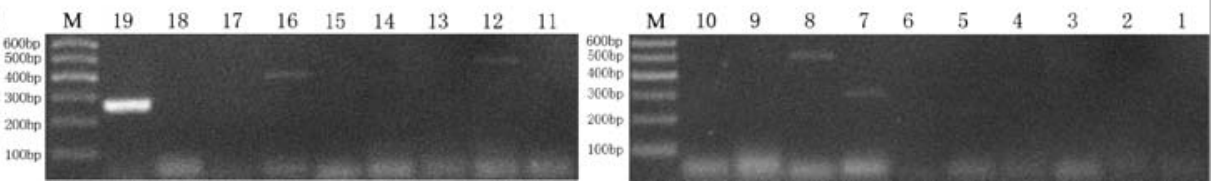

Figure 2. Different PDE subtypes mRNA was detected in skeletal muscle primary cultured cells using 1:50 (a), 1:100 (b) and 1:1000 (c) dilution of cDNA reaction mixture as PCR template. Lanes from 1 to 18 correspond to PDE1A-PDE11A; lane 19 corresponds to control GAPDH. Lane M is molecular weight markers 
RT-PCR was used to detect the mRNA transcripts of all PDE isozymes except PDE6 in mouse skeletal muscle tissue and primary cultured myocytes. A similar expression profile of PDE subtype mRNAs was shown in skeletal muscle tissue and primary cultured myocytes. All PDE subtypes except PDE8B showed specific bands after PCR amplification, as expected (Figures 1a-b). PDE4A, 4B, PDE7A, PDE9A and PDE10A signals were strong, while PDE1C was barely detected. Most PCR signals were stronger in cultured myocytes than in tissue. Furthermore, with diluting the myocyte cDNA reaction mixture, less specific bands and weaker signal intensity were observed (Figures 2a-c). When diluted by 1:1000, only PDE4A, PDE4B, PDE7A, and PDE9A subtypes were detected with very weak signals. Among these dilutions PDE4A and 4B subtype PCR signals were always stronger than the other detected ones.

\section{DISCUSSION}

In the present study, we demonstrate for the first time the presence of mRNA for all PDE subtypes except PDE6A-6D in mouse skeletal muscle tissue and primary cultured myocytes. The results show that skeletal muscle tissue and myocytes expressed seventeen PDE subtype isozyme mRNA with different intensities except PDE8B. The PDE mRNA expression profile in skeletal muscle tissue is approximately the same as in primary cultured myocytes, but almost always stronger expression is shown in myocytes. This might be related to: 1 . RNA extract in myocytes being purer than in skeletal muscle tissue, and 2. primary cultured myocytes possess a similar property with animal postnatal skeletal muscle, in which the amount of muscle cells does not change, but only their size is increasingly larger. In primary cultured myocytes, once they differentiate and myotubes fuse, cells no longer proliferate. At this phase, myotubes can be regarded as postnatal skeletal muscle. Thus, primary cultured myocytes are more suitably used to observe the expression of PDE isozymes in skeletal muscle.

Using different dilutions of cDNA reaction mixtures as templates for PCR amplification showed that PDE4A, 4B, PDE7A and PDE9A concentrations are high in skeletal muscle; PDE2A, PDE3A, PDE4D, PDE7B, PDE10A concentrations are moderate; and the remaining ones are low. Some results were consistent with previous findings, which showed that PDE4B and PDE7A were abundantly expressed in human and mouse skeletal muscle (Han et al., 1997; Bingham et al., 2006) and that PDE7B was moderately expressed in human and rat skeletal muscle (Sasaki et al., 2002). However, contrary to a previous report (Soderling et al., 1998), PDE9A was highly expressed in skeletal muscle in our study. PDE9A selectively hydrolyses cGMP, so it is suggested that PDE9A is the main hydrolytic enzyme for cGMP, which is distributed in very low levels 
in skeletal muscle (Zan et al., 1999). PDE8B was not expressed in either ICR mouse skeletal muscle tissue or myocytes. However, it was moderately expressed in Chinese experimental small pig skeletal muscle tissue in our previous study. It is suggested that PDE8B is not involved in cAMP hydrolysis in ICR mouse skeletal muscle.

PDE isozymes are of growing interest as targets for therapeutic intervention. Therefore, in context with the complexity of the PDE gene families, it will be of increasing significance to establish precisely which of the many different PDE types/subtypes are expressed in a given cell type. The PDE4 family is one of the most extensively studied PDE isozymes. Expression has been demonstrated in vascular, T-cell, various inflammatory cells as well as in airway smooth muscle cells, and the potential use of specific type 4 PDE inhibitors alone or in combination with prostaglandin therapy has been explored to abrogate inflammation in pulmonary vascular disorders (Wagner et al., 1997; Gao et al., 1998). Moreover, it has been reported that PDE7A mRNA is present in primary epithelial cells (Gantner et al., 1998) and human peripheral blood T lymphocytes (Giembycz et al., 1996) and B lymphocytes (Gantner et al., 1998). Using antisense oligonucleotides, a role for PDE7A in T cell proliferation and interleukin (IL)-2 production (Li et al., 1999) has been indicated. But, compared with PDE4 and PDE7, little is known of the expression and functions of PDE9A in various tissues and cells. Because pharmacologic targeting of specific PDE isozymes is possible, it will be of interest to determine how these PDE isozymes influence skeletal muscle biological processes. In addition, it is important due to the potential of using PDE isozymes inhibitors to accelerate the growth rate of animals.

Selection for increased growth rate in farm and laboratory animals has been used to develop lines with increased body and muscle weights. Growth rate and muscle protein turnover are closely linked. Altered rates of protein synthesis and degradation can greatly affect the rate of protein accumulation, therefore, change animal growth rates. Numerous studies have shown that the administration of $\beta$-adrenergic agonists modulates growth by increasing skeletal muscle mass in many species of animals (Yang and McElligott, 1989; Mersmann, 1998). In general, these agents enhance the rate of gain, decrease feed consumption, increase the amount of skeletal muscle tissue, and decrease the amount of adipose tissue (Mersmann, 1995). It is well known that they exert their effects through the $\beta$-adrenergic-mediated increase in intracellular cAMP and subsequent activation of specific protein kinases. Also, increased intracellular levels of cAMP inhibit skeletal muscle alanine and glutamine formation and release, and increased intracellular levels of cGMP stimulate skeletal muscle proteolysis and the formation and release of the amino acids alanine and glutamine (Garber, 1978; Garber et al., 1980). Although the function of the cyclic nucleotides in skeletal muscle involved in protein metabolism, which intervenes in the growth of skeletal muscle, is well 
known, the role of PDE in increasing muscle weight is little understood. Moreover, which PDE isozymes mainly contribute to the function and how are not known in detail. However, it had been reported that a xanthine derivative, isobutylmethylxanthine (IBMX), which is an nonspecific inhibitor of PDE isozymes, inhibits PDEs and increases the intracellular concentration of cAMP in skeletal muscle, reduces proteolysis in both rat soleus and extensor digitorum longus muscles (Navegantes et al., 2000). Thus, this result suggests that PDE isozymes play an important role in skeletal muscle protein metabolism during biological development.

Our results show that almost all PDE isozyme mRNAs are expressed in skeletal muscle, and that PDE4A, 4B, PDE7A and PDE9A predominate. Thus, we deduce that PDE4A, 4B, PDE7A and PDE9A play a key role in protein turnover in skeletal muscle. However, we assume that the intracellular system for hydrolysis of the second messenger cAMP and cGMP is more complex than currently postulated because there is abundant cross talk between different PDE isozymes.

\section{CONCLUSIONS}

We studied expression of PDE isozymes using RT-PCR and found a similar pattern of mRNA transcript expression in skeletal muscle tissue and primary cultured myocytes. The results suggest that PDE could be involved in multiple biological processes in skeletal muscle through their ability to modulate cAMP and cGMP levels. Thus, the detection of expression of PDE in skeletal muscle was the foundation for selection of PDE inhibitors for regulation of animal growth and the functions of PDE isozymes in skeletal muscle require further investigation.

\section{REFERENCES}

Bingham J., Sudarsanam S.B., Srinivasan S., 2006. Profiling human phosphodiesterase genes and splice isoforms. Biochem. Biophys. Res. Commun. 350, 25-32

Cheng C.Y., Boettcher B., 1982. Partial characterization of human spermatozoal phosphodiesterase and adenylate cyclase and the effect of steroids on their activities. Int. J. Androl. 5, 253-266

Dumont J.E., Jauniaux J.C., Roger P.P., 1989. The cyclic AMP-mediated stimulation of cell proliferation. Trends Biochem. Sci. 14, 67-71

Gantner F., Gotz C., Gekeler V., Schudt C., Wendel A., Hatzelmann A., 1998. Phosphodiesterase profile of human $\mathrm{B}$ lymphocytes from normal and atopic donors and the effects of PDE inhibition on B cell proliferation. Brit. J. Pharmacol. 123, 1031-1038

Gao Y., Tolsa J.F., Shen H., Raj J.U., 1998. Effect of selective phosphodiesterase inhibitors on response of ovine pulmonary arteries to prostaglandin E2. J. Appl. Physiol. 84, 13-18

Garber A.J., 1978. The regulation of skeletal muscle alanine and glutamine formation and release in experimental chronic uremia in the rat: subsensitivity of adenylate cyclase and amino acid release to epinephrine and serotonin. J. Clin. Invest. 62, 633-641 
Garber A.J., Birnbaumer L., Bornet E.P., Thompson W.J., Entman M.L., 1980. Skeletal muscle protein and amino acid metabolism in hereditary mouse muscular dystrophy. The role of disordered cyclic nucleotide metabolism in the accelerated alanine and glutamine formation and release. J. Biol. Chem. 255, 8325-8333

Giembycz M.A., Corrigan C.J., Seybold J., Newton R., Barnes P.J., 1996. Identification of cyclic AMP phosphodiesterases 3, 4 and 7 in human CD4+ and CD8+ T-lymphocytes: role in regulating proliferation and the biosynthesis of interleukin-2. Brit. J. Pharmacol. 118, 1945-1958

Han P., Zhu X., Michaeli T., 1997. Alternative splicing of the high affinity cAMP-specific phosphodiesterase (PDE7A) mRNA in human skeletal muscle and heart. J. Biol. Chem. 272, 1615216157

Hellsten Y., Frandsen U., 1997. Adenosine formation in contracting primary rat skeletal muscle cells and endothelial cells in culture. J. Physiol. 504, 695-704

Kuthe A., Wiedenroth A., Magert H.J., Uckert S., Forssmann W.G., Stief C.G., Jonas U., 2001. Expression of different phosphodiesterase genes in human cavernous smooth muscle. J. Urol. $165,280-283$

Li L., Yee C., Beavo J.A., 1999. CD3- and CD28-dependent induction of PDE7 required for T cell activation. Science 283, 848-851

Manganiello V.C., Murata T., Taira M., Belfrage P., Degerman E., 1995. Diversity in cyclic nucleotide phosphodiesterase isoenzyme families. Arch. Biochem. Biophys. 322, 1-13

Mersmann H.J., 1995. Species variation in mechanisms for modulation of growth by $\beta$-adrenergic receptors. J. Nutr. 125, 1777S-1782S

Mersmann H.J., 1998. Overview of the effects of $\beta$-adrenergic receptor agonists on animal growth including mechanisms of action. J. Anim. Sci. 76,160-172

Navegantes L.C., Resano N.M., Migliorini R.H., Kettelhut I.C., 2000. Role of adrenoceptors and cAMP on the catecholamine-induced inhibition of proteolysis in rat skeletal muscle. Amer. J. Physiol.-Endocrinol. Met. 279, E663-E668

Sasaki T., Kotera J., Omori K., 2002. Novel alternative splice variants of rat phosphodiesterase 7B showing unique tissue-specific expression and phosphorylation. J. Biol. Chem. 361, 211-220

Shaulsky G., Fuller D., Loomis W.F., 1998. A cAMP-phosphodiesterase controls PKA-dependent differentiation. Development 125, 691-699

Soderling S.H., Bayuga S.J., Beavo J.A., 1998. Identification and characterization of a novel family of cyclic nucleotide phosphodiesterases. J. Biol. Chem. 273, 15553-15558

Soderling S.H., Beavo J.A., 2000. Regulation of cAMP and cGMP signaling: new phosphodiesterases and new functions. Curr. Opin. Cell Biol. 12, 174-179

Wagner R.S., Smith C.J., Taylor A.M., Rhoades R.A., 1997. Phosphodiesterase inhibition improves agonist-induced relaxation of hypertensive pulmonary arteries. J. Pharmacol. Exp. Ther. 282, 1650-1657

Yang Y.T., McElligott M.A., 1989. Multiple actions of $\beta$-adrenergic agonists on skeletal muscle and adipose tissue. Biochem. J. 261, 1-10

Zan L.S., Lü J.Y., Ma Z.K., 1999. Distribution of endogenous CNT in animal (in Chinese). J. Nucl. Agr. Sci. 13, 343-346 